\title{
LOS TITULARES DE LA INICIATIVA DEL PROCESO AUTONOMICO
}

\author{
353.072 .01 \\ por \\ Enrique Alvarez Conde
}

SUMARIO: I. INTRODUCCION.-II. REGULACION CONSTITUCIONAL DE LA INICIACION DEL PROCESO AUTONOMICO.-III. EL SU. PUESTO GENERAL.-IV. SUPUESTOS ESPECIALES: 1. LAS CORTES GENERALES COMO TITULARES DE LA INICIATIVA AUTONOMICA. 2. LOS ORGANOS PREAUTONÓMICOS COMO TITULARES DE LA INICIATIVA AUTONÓMICA. 3. LAS DIPUTACIONES, LOS ORGANOS INTERINSULARES Y LOS MUNICIPIOS COMO TITULARES ESPECIALES DE LA INICIATIVA AUTONOMICA.-V. SUPUESTOS PARTICULARES: 1. LA INICIATIVA AUTONOMICA EN EL CASO DE NavarRa. 2. La iniciativa aUtonómica en los casos de CeUta Y MeLILLA.-VI. LA POSTURA DE LAS FUERZAS POLITICAS.

\section{INTRODUCCION}

El conjunto de trámites, plazos y normas diversas hacia la creación de una Comunidad autónoma constituye lo que se ha denominado el proceso autonómico, que, como todo proceso, tiene un comienzo y un final. El comienzo de este proceso se produce cuando los titulares, constitucionalmente reconocidos, ejercen el derecho a la iniciativa autonómica. Su final, cuando queda institucionalizada la autonomía de la Comunidad. Entre el comienzo y el fin existen una serie de cuestiones fundamentales, siendo la más importante la elaboración y aprobación del Estatuto de Autonomía (1).

Este trabajo únicamente va a versar sobre el inicio del proceso autonómico, es decir, sobre la problemática de los titulares de la

(1) En torno al proceso autonómico, cfr. Ricardo MEDINA: aEl proceso autonómico. La iniciativa», ponencia presentada a las IV Jornadas Internacionales de Ciencia Política y Desarrollo Constitucional, celebradas en Valencia en octubre de 1978. 
iniciativa autonómica. A este respecto, distinguiremos entre el supuesto general, que es el reconocido en el artículo 143, 2, de la Constitución; los supuestos especiales, donde analizaremos la iniciativa autonómica de las Cortes, de los órganos preautonómicos y de las Corporaciones locales establecidas en el artículo 151, 1, de la misma, y, finalmente, los supuestos particulares, que se refieren a los casos de Navarra, Ceuta y Melilla. Pero antes, expongamos, brevemente, el distinto tratamiento que ha recibido esta cuestión a lo largo del "proceso constituyente».

\section{REGULACION CONSTITUCIONAL DE LA INICIACION DEL PROCESO AUTONOMICO}

En el primer dictamen de la ponencia, la iniciativa autonómica, como supuesto general, estaba reconocida a los Ayuntamientos, estableciéndose, además, como supuestos especiales, que dicha iniciativa fuese ejercida por las Cortes Generales y por los órganos preautonómicos, no contemplándose, pues, la existencia de lo que nosotros hemos denominado supuestos particulares (2).

En el segundo dictamen de la ponencia, de 12 de abril, se establece, como supuesto general, que la iniciativa autonómica corresponde a las Diputaciones, órganos insulares correspondientes y $\mathrm{Mu}$ nicipios. Asimismo, como supuesto especial, se atribuía dicha iniciativa a las Cortes Generales y, como supuesto particular, se contemplaba el caso de Navarra. Así pues, este segundo dictamen supone una profunda modificación en relación con el anterior, desapareciendo algunos titulares de la iniciativa autonómica, como son los órganos preautonómicos, y apareciendo otros, como las Diputaciones, órganos insulares y contemplándose el caso particular de Navarra (3).

El dictamen de la Comisión del Congreso configura, como en tantas otras cuestiones, de una forma prácticamente ya definitiva, los titulares de la iniciativa autonómica. Como supuesto general, los titulares son las Diputaciones u órganos interinsulares y los Municipios. Como supuestos especiales, tenemos a las Cortes Generales, órganos preautonómicos y a los Municipios con determi-

(2) Vid. el artículo 129 y la disposición transitoria tercera del primer dictamen de la ponencia en Boletín Oficial de las Cortes -en adelante B. O. C.-, núm. 44, de 5 de enero.

(3) Vid. los artículos 146, 140 y disposición transitoria tercera del dictamen en $B$. O. C., núm. 82 , de 17 de abril. 
nados requisitos. Finalmente, como supuestos particulares, se contemplan los casos de Navarra, Ceuta y Melilla (4).

El texto aprobado por el Pleno del Congreso no presenta ninguna modificación relevante (5). Por su parte, el texto adoptado por la Comisión del Senado respeta los titulares consagrados en los supuestos generales y particulares, con algunas modificaciones en cuanto al procedimiento y trámites, introduciendo, en los supuestos especiales, a las Diputaciones como titulares de la iniciativa autonómica (6). El Pleno del Senado no introduce modificación alguna, presentando únicamente algunas variaciones sistemáticas y procedimentales (7). Y, finalmente, el dictamen de la Comisión Mixta tampoco altera sustancialmente la cuestión (8).

Este es, a grandes rasgos, el tratamiento que ha experimentado el problema que nos ocupa en las diferentes redacciones del texto constitucional. Como hemos visto, los titulares de la iniciativa autonómica aparecen ya prácticamente configurados por el dictamen de la Comisión del Congreso, con la modificación introducida por la Comisión del Senado referente a las Diputaciones. No hemos efectuado un análisis pormenorizado de su diversa regulación, sino tan sólo una enumeración, pues creemos que, desde un punto de vista sistemático, es mejor hacer dicho análisis sobre el texto definitivo de la Constitución, que se verá, además, complementado con el análisis de las posturas de las diversas fuerzas políticas parlamentarias.

\section{EL SUPUESTO GENERAL}

Viene establecido en el artículo 143: «2. La iniciativa del proceso autonómico corresponde a todas las Diputaciones interesadas o al órgano interinsular correspondiente y a las dos terceras partes de los Municipios cuya población represente, al menos, la mayoría

(4) Vid. los artículos 137, 142, 145 y disposiciones transitorias primera, cuarta y quinta del dictamen de la Comisión del Congreso en $B . O$. C., núm. 121, de 1 de julio.

(5) Vid. los mismos artículos de la nota anterior en texto aprobado por el Pleno del Congreso en B. O. C., núm, 135, de 24 de julio.

(6) Vid. artículos 142,147,150 y disposiciones transitorias primera, cuarta y quinta del dictamen de la Comisión del Senado en B. O. C., núm. 157, de 6 de octubre.

(7) Vid. los mismos artículos en texto aprobado por el Pleno del Senado en Boletin Oficial de las Cortes, núm. 161, de 13 de octubre.

(8) Vid. artículos 143,144,151 y dísposiciones transitorias primera, cuarta y quinta del dictamen de la Comisión Mixta, que coincide con la definitiva redacción del texto constitucional, de 28 de octubre. 
del censo electoral de cada Provincia o isla. Estos requisitos deberán ser cumplidos en el plazo de seis meses desde el primer acuerdo adoptado al respecto por alguna de las Corporaciones locales interesadas. 3. La iniciativa, en caso de no prosperar, solamente podrá reiterarse pasados cinco años». Esta redacción plantea una serie de cuestiones fundamentales, que se refieren a los propios titulares de la iniciativa autonómica, a los requisitos exigidos y a los plazos establecidos para su ejercicio.

Los titulares de la iniciativa autonómica son, pues, las Diputaciones, los órganos interinsulares correspondientes y los Municipios. En principio, parece existir aquí una grave incorrección terminológica y sistemática, pues las Diputaciones, como señala el artículo 141, 2, no son Entidades locales con personalidad jurídica propia, sino el órgano de administración y gobierno de la Provincia, la cual sí tiene personalidad jurídica propia. Por el contrario, el Municipio sí es una Entidad local con personalidad jurídica propia (art. 140). Resulta, pues, contradictorio que se hable de Diputaciones y Municipios como sujetos de la iniciativa autonómica. Lo más correcto hubiera sido hablar de Provincias y Municipios o, en su caso, de Diputaciones y Ayuntamientos.

Pero, sin duda, la cuestión más importante es el determinar si esta iniciativa, para que prospere, puede ser realizada por cualquiera de sus titulares o, necesariamente, tiene que se realizada por todos ellos. En principio, el artículo 143, 2, parece exigir que la iniciativa sea ejercitada tanto por unos como por otros. No otro sentido tiene el empleo de la conjunción "y»-que tiene aquí un carácter eminentemente copulativo y no disyuntivo-, que implica la configuración de una iniciativa cumulativa. Es decir, para que dicha iniciativa prospere debe ser ejercida por todos los titulares mencionados y en los plazos y con los requisitos establecidos. Otro sentido hubiera tenido el precepto si en vez de emplearse la conjunción "y» se utilizase la conjunción "o», tal y como se recogía en el artículo 146 del dictamen de la ponencia de 17 de abril, en cuyo caso se trataría de dos iniciativas autonómicas diferentes y alternativas y no meramente cumulativas. De todas formas, la redacción del precepto no es muy adecuada y, quizás, hubiera sido mucho más nítida si se hubiese utilizado una redacción semejante a la del artículo 151, 1, que no deja lugar a ningún tipo de duda.

Se trata, por tanto, de una iniciativa cumulativa. Además, ésta es la postura que se deduce de los debates parlamentarios en tor- 
no a esta cuestión, donde también se planteó el problema (9). Con ello, evidentemente, se obstaculiza la iniciativa del proceso autonómico, razón por la cual no debería haberse concebido una iniciativa cumulativa, sino alternativa.

En cuanto a los requisitos establecidos para ejercer dicha iniciativa, la Constitución no dice nada de cómo ha de ejercerse, de si ha de hacerse por acuerdo de los titulares autonómicos, o por sus representantes o Presidentes, ni qué tipo de cualificación se exige. Ante el silencio constitucional, en principio hay que excluir tanto a los Presidentes de Diputaciones como a los Alcaldes, pues únicamente se habla de Diputaciones y Municipios, es decir, de órganos colegiados. Incluso podría plantearse el problema, en el caso de los Municipios, de si dicha iniciativa corresponde a la totalidad del Municipio (bien a sus habitantes o a la multiplicidad de órganos existentes) o únicamente a sus órganos de gobierno y administración (art. 140), es decir, a los Ayuntamientos. Esta última parece ser la solución correcta y la que estaba en la mente de los "constituyentes», pero que no queda establecida de una forma taxativamente clara. Por otra parte, tampoco se habla de cómo se ha de ejercer dicha iniciativa, si ha de ser por acuerdo simple o por acuerdo de mayoría absoluta, $u$ otra cualificación cualquiera. Asimismo, tampoco se establece si esa hipotética cualificación para adoptar el acuerdo ha de hacerse por mayoría de los presentes o de los miembros de las citadas Corporaciones.

En estas cuestiones, la Constitución únicamente habla de «Diputaciones interesadas», lo cual parece querer indicar que puede haber, dentro del territorio que pretenda convertirse en Comunidad autónoma, Diputaciones que no estén interesadas y que, por lo tanto, no tengan por qué ser titulares de la iniciativa autonómica. También se habla de "las dos terceras partes de los Municipios cuya población represente, al menos, la mayoría del censo electoral de cada Provincia o isla». Ello supone otorgar un derecho de veto a la tercera parte de los Municipios más uno, con independencia de la población que representen. Pero, además, parece existir una contradicción entre los términos población y censo electoral. En efecto, puede interpretarse que el término población incide en

(9) Cfr. especialmente los debates en el Pleno del Congreso sobre el antiguo artículo 137. Son, a este respecto, significativos los discursos de los señores Gómez de las Roces, Gastón y Bermúdez (D. S. Congreso, sesión del 18 de julio, págs. 4388 y siguientes). 
que las dos terceras partes de los Municipios exigidos se refiere, más que a estas Entidades locales, a la población que dichos $\mathrm{Mu}$ nicipios representan. Hasta aquí la redacción parece lógica y convincente. Pero sucede que al hablar de población, ésta no viene referida a la totalidad de los habitantes de los Municipios respectivos, sino al censo electoral de cada Provincia o isla. Es decir, no se trata de la mayoría - se entiende necesariamente relativa- de la población de cada Provincia o isla, sino de la mayoría relativa del censo electoral. Con ello, se excluye automáticamente a los menores de dieciocho años, que no son computados en este caso. Incluso pudiera pensarse que, al no especificarse de qué censo electoral se trata, pues puede haber en cada Provincia o isla diferentes categorías o modalidades de censo electoral, que el tope no sean los dieciocho años, sino aún mayor. De todas formas, creo que ésta es una interpretación un. tanto forzada y que debe admitirse la solución anterior. Aun así, resulta evidente que no se trata de la mayoría relativa de la población, como sería de desear, sino del censo electoral, excluyéndose a los menores de dieciocho años y a los que no tengan adquirida vecindad en la Provincia o isla.

Por otra parte, hemos de señalar que no se trata de las dos terceras partes de los Municipios de la totalidad del territorio afectado, sino de las dos terceras partes de los Municipios de cada Provincia o isla. Con ello, por una parte, se potencia notoriamente a estas Entidades y, por otra, puede suceder que las Provincias o islas, fundamentalmente estas últimas, menos pobladas, boicoteen la iniciativa autonómica. Por ello, creo que esta exigencia debería haber sido suprimida y referirse únicamente a las dos terceras partes de los Municipios de la totalidad del territorio que pretenda convertirse en Comunidad autónoma.

Finalmente, el precepto establece una serie de plazos, dentro de los cuales deben cumplirse estos requisitos. En principio, señala que deben ser cumplidos dentro de seis meses a contar desde que se produzca el primer acuerdo de alguna de las Corporaciones locales interesadas. Es decir, que desde el primer acuerdo adoptado por alguna de las Corporaciones locales hasta el total cumplimiento de los requisitos establecidos para el ejercicio de la iniciativa autonómica, han de transcurrir tan sólo seis meses. Este plazo, aparte de que los Municipios no tienen la consideración de Corporaciones locales, es a todas luces corto e insuficiente y puede dar lugar a que en determinado Ayuntamiento 
- Diputación, con intención de boicotear, en algún modo, la iniciativa autonómica, se adelante en su acuerdo y frene, con ello, el proceso autonómico, porque, como señala el artículo 143,3 , si la iniciativa autonómica, con los requisitos anteriormente mencionados, no prosperase, únicamente podría ser reiterada pasados cinco años. Es decir, con el acuerdo de una sola Corporación local puede boicotearse toda la iniciativa autonómica. Piénsese, además, que estamos en presencia de una iniciativa cumulativa y que se requiere el acuerdo de muchas Corporaciones en el plazo exiguo de seis meses.

Acabamos de decir que este plazo de seis meses empieza a contar -término a quo- desde el momento en que se produzca el primer acuerdo de una de las Corporaciones locales. Sin embargo, la disposición transitoria tercera señala que «la iniciativa del proceso autonómico por parte de las Corporaciones locales o de sus miembros, prevista en el apartado 2 del artículo 143, se entiende diferida, con todos sus efectos, hasta la celebración de las primeras elecciones locales una vez vigente la Constitución». Esta posposición del ejercicio de la iniciativa autonómica puede justificarse en base a que los actuales Ayuntamientos y Diputaciones carecen del más elemental carácter democrático, tal como parecen exigir los artículos 140 y siguientes de la Constitución, y, por consiguiente, es necesario esperar a su renovación mediante elecciones libres. Sin embargo, la redacción de esta disposición transitoria plantea serios problemas.

En principio, parece atribuirse la iniciativa autonómica no solamente a las Corporaciones locales mencionadas, sino también a sus miembros, como pudieran ser, por ejemplo, el Presidente de la Diputación, el Alcalde, los diputados provinciales y los concejales. Ello supone una absoluta incorrección que puede plantear graves problemas y dificultar o boicotear de un modo absoluto la iniciativa autonómica. La introducción de las palabras "O de sus miembros» no admite justificación alguna y sería lamentable que se tratase de lapsus, por razón de tiempo, de nuestros «constituyentes». Y es que la cuestión trasciende del mero error terminológico, máxime si interpretamos que el plazo de seis meses establecido en el artículo 143, 2, puede empezar a contarse no desde el acuerdo adoptado por una de las Corporaciones locales, sino desde el acuerdo o propuesta de alguno de los miembros de las citadas Corporaciones.

Además, esta disposición transitoria puede plantear otras cues- 
tiones. Así, la iniciativa autonómica queda diferida hasta la «celebración" de las primeras elecciones locales. Con ello, pudiera señalarse que por celebración se entiende el día que se efectúan las correspondientes elecciones. $\mathrm{Y}$ a partir de ese día ya puede iniciarse el proceso autonómico. De esta forma, puede quedar totalmente desvirtuado el sentido de esta disposición transitoria, pues al día siguiente de la celebración de las elecciones aún no se habrán computado todos los votos, aún no se sabrá la composición de los Ayuntamientos y Diputaciones y, finalmente, aún éstos no se habrán constituido. Así pues, los Ayuntamientos o Diputaciones anteriores, que aún continúen en sus funciones hasta la constitución definitiva de los resultantes de las elecciones, podrían ejercer dicha iniciativa autonómica. Por ello, creo que hubiera sido necesario señalar que la iniciativa se entendía diferida hasta la constitución de los nuevos Ayuntamientos y Diputaciones resultantes de la celebración de las primeras elecciones locales, una vez vigente la Constitución.

Por último, hemos de señalar que este supuesto general de la iniciativa autonómica parece concebido para aquellas Comunidades autónomas no privilegiadas, sin que, por otra parte, esté excluido para otra categoría de Comunidades autónomas, tema sobre el cual elaboraremos otro trabajo.

\section{SUPUESTOS ESPECIALES}

Dentro de este apartado vamos a estudiar a aquellos titulares de la iniciativa autonómica que pueden sustituir la iniciativa de las Diputaciones y Municipios, como son las Cortes Generales y los órganos preautonómicos, y también la propia iniciativa de Municipios y Diputaciones, tal y como la configura el artículo 151, 1. Distinguiremos, pues, tres apartados diferentes.

\section{Las'Cortes Generales como titulares de la iniciativa AUTONOMICA}

El artículo 144 establece: «Las Cortes Generales, mediante Ley Orgánica, podrán por motivos de interés nacional: a) Autorizar la constitución de una Comunidad autónoma cuando su ámbito territorial no supere el de una Provincia y no reúna las condiciones del apartado 1 del artículo 143; b) Autorizar a acordar, en su caso, un 
Estatuto de Autonomía para territorios que no estén integrados en la organización provincial; c) Sustituir la iniciativa de las Corporaciones locales a que se refiere el apartado 2 del artículo 143 ». Vemos, pues, que esta titularidad de las Cortes Generales se establece con carácter potestativo -el texto habla de "podrán»- y que tiene que realizarse conjuntamente por el Senado y el Congreso, y no separadamente. Unicamente se puede ejercer esta iniciativa por motivos de interés nacional, expresión ésta un tanto vaga, motivos que deben ser apreciados exclusivamente por las Cortes Generales. El procedimiento para ejercer esta iniciativa es mediante una Ley Orgánica, para cuya aprobación, modificación o derogación se exige mayoría absoluta del Congreso en una votación final sobre el conjunto del proyecto (art. 81,2 ). Con ello, la titularidad de la iniciativa corresponde, de facto, al Congreso de los Diputados, aunque también el Senado participe, si bien no al mismo nivel, de dicha iniciativa, pues el Congreso por sí solo no puede ejercerla. Analicemos ahora cada uno de estos supuestos para determinar si en todos ellos se trata de una auténtica iniciativa autonómica.

a) En el primer apartado se atribuye a las Cortes la facultad de autorizar la constitución de una Comunidad autónoma cuando se produzcan las dos siguientes circunstancias: a) Que su ámbito territorial no supere el de una Provincia, y $b$ ) Que no reúna las condiciones establecidas en el artículo 143, 1. Es decir, que las Cortes Generales únicamente pueden autorizar a aquellos territorios cuya extensión sea igual o menor a la de una Provincia y que, además, ese territorio no tenga entidad regional histórica, circunstancia ésta que también deberá ser apreciada por la Cortes Generales. Este apartado parece pensado para los supuestos de Ceuta y Melilla, sobre los cuales ya volveremos cuando analicemos la disposición transitoria quinta, y para los casos concretos de León, Logroño, Santander e incluso Navarra, si no decidiera integrarse en el País Vasco tal y como establece la disposición transitoria cuarta, siempre en el hipotético caso de que se considerase que estas Provincias carecen de entidad regional histórica, pues en caso contrario estarían comprendidas en el artículo 143, 1, y las Cortes ya no podrían extender su autorización, ya que se encontrarían en el supuesto general, anteriormente analizado.

También se nos plantea el problema de si, en este supuesto, las Cortes son auténticos titulares o no de la iniciativa autonómica. El precepto habla de «autorizar», lo cual implica que los titulares de 
la iniciativa autonómica son otros y que las Cortes tienen que autorizar dicha iniciativa. Esta es la interpretación correcta teniendo en cuenta, como luego veremos, lo dispuesto en la disposición transitoria quinta, en la cual la iniciativa corresponde a los Ayuntamientos de Ceuta y Melilla y a las Cortes la referida autorización. También hay que señalar que si bien las Cortes no son propiamente titulares de la iniciativa autonómica, sin embargo, al ser necesaria su autorización, su papel en el proceso autonómico tiene especial trascendencia, requiriéndose para el inicio de dicho proceso dos presencias: la de los titulares de la iniciativa autonómica y la de las Cortes autorizando dicha iniciativa.

b) En el segundo supuesto, el planteamiento es muy distinto. En principio, las Cortes pueden «autorizar o acordar». Si únicamente autorizan, tampoco son los titulares de la iniciativa autonómica, encontrándonos en la misma situación que en el supuesto anterior. Pero si las Cortes no autorizan, sino que acuerdan, entonces sí que son los auténticos titulares de la iniciativa autonómica.

Sin embargo, este supuesto es radicalmente distinto del anterior. En principio, las Cortes no autorizan o acuerdan la constitución de una Comunidad autónoma, sino un Estatuto de Autonomía, lo cual no implica necesariamente la futura existencia de una Comunidad autónoma. El ámbito territorial sobre el que se ha de producir dicha autorización o acuerdo son aquellos territorios que no están integrados en la organización provincial. Ello implica que este supuesto únicamente es aplicable al futuro de Gibraltar, pues es la única parte del territorio no integrada en la organización provincial, ya que Ceuta y Melilla sí lo están. Si bien esta solución puede ser loable, pues en algún modo se prevé la futura situación de Gibraltar, sin embargo plantea algún problema. Al no hablarse de Comunidad autónoma y sí de Estatuto de Autonomía, parece indicar que Gibraltar puede llegar a tener una situación muy particular dentro de la organización territorial española. Esto, sin embargo, no puede ser así, pues el artículo 137 señala que «el Estado se organiza territorialmente en Municipios, Provincias y en las Comunidades autónomas que se constituyan". Con ello, necesariamente Gibraltar habrá de adoptar una de estas categorías territoriales. Seguramente, y para conceder una mayor libertad de opción a los gibraltareños, es por lo que en el precepto no se habla de Comunidad autónoma, sino simplemente de Estatuto de Autonomía. A ello pudiera objetarse que Gibraltar necesariamente tendrá que adop- 
tar la condición de Comunidad autónoma, pues la Constitución no pre'ré, para los Municipios y Provincias, la existencia de un Estatuto de Autonomía, aunque diga que estas Entidades gozan de autonomía (arts. 137 y sigs.), y, sin embargo, sí que señala, en el artículo 147, 1, que los Estatutos serán la norma institucional básica de cada Comunidad autónoma.

De todas formas, es loable esta previsión que en principio puede ser compatible con una amplia autonomía, sin duda muy necesaria, para los gibraltareños, máxime si las Cortes se limitan a autorizar dicho Estatuto de un modo meramente formal.

c) Finalmente, en el tercer supuesto se contempla a las Cortes como auténticos titulares de la iniciativa autonómica, al poder sustituir la iniciativa de las Diputaciones y Municipios prevista en el artículo 143, 2. Aquí no son aplicables los plazos y requisitos estudiados en el supuesto general, bastando un acuerdo de las Cortes, que formalmente tiene que ser una Ley Orgánica, producido por motivos de interés nacional.

Esta facultad atribuida a las Cortes puede encerrar ciertos peligros. En efecto, pudiera parecer que el territorio afectado dispondría de una autonomía no originaria ni reconocida, someramente concedida por un acuerdo de las Cortes Generales. Por otra parte, pueden producirse conflictos entre las Cortes Generales y las Corporaciones locales de los territorios afectados. Sin embargo, también puede producir efectos positivos en el supuesto, por ejemplo, de que alguna de las Corporaciones citadas boicotease la iniciativa del proceso autonómico, lo cual -como hemos visto- es relativamente fácil. Se trata, en suma, de un arma de doble filo, que necesitará una prudente aplicación, caso de que ésta se produzca, y que en todo caso requerirá una adecuada interpretación, con intervención incluso del futuro Tribunal Constitucional, de esos «motivos de interés nacional».

\section{LOS ORGANOS PREAUTONÓMICOS COMO TITULARES DE LA INICIATIVA AUTONOMICA}

La disposición transitoria primera establece: «En los territorios dotados de un régimen provisional de autonomía, sus órganos colegiados superiores, mediante acuerdo adoptado por la mayoría absoluta de sus miembros, podrán sustituir la iniciativa que el apartado 2 del artículo 143 atribuye a las Diputaciones provinciales o 
a los órganos interinsulares correspondientes». Esta redacción nos plantea una serie de cuestiones.

En primer lugar, se habla de «territorios dotados de un régimen provisional de autonomía», lo cual implica que estos territorios pueden considerarse autónomos, aunque provisionalmente. Ello sería cierto si las funciones y competencias de los órganos representativos de esos territorios fueran susceptibles de ser calificadas de autónomas, entendiendo por autonomía el ejercicio, material y formal, de la potestad legislativa y, en sentido más amplio, identificándolo con autogobierno. Sin embargo, ni los entes preautonómicos tienen potestades legislativas ni puede afirmarse que sean unos entes de autogobierno. Sus facultades son meramente administrativas y de gobierno interior.

En segundo lugar, hemos de señalar que los titulares de la iniciativa autonómica son los órganos colegiados superiores de los entes preautonómicos — sus Consejos o Juntas, según los casos-, y no los propios entes preautonómicos. Además se trata de una función potestativa —el texto habla de "podrán sustituir»- que no tiene por qué necesariamente realizarse y que, en todo caso, requiere el acuerdo de la mayoría absoluta de los miembros de los citados órganos, excluyéndose tanto al Presidente de la entidad preautonómica como a otros posibles órganos colegiados existentes.

Finalmente, el último inciso de esta disposición transitoria nos plantea una serie de cuestiones. En principio, los mencionados órganos colegiados superiores sólo pueden sustituir la iniciativa de las Diputaciones u órganos interinsulares correspondientes. Sin embargo, la iniciativa autonómica (art. 143, 2) también corresponde, como hemos visto, a los Municipios, a los cuales no pueden susti. tuir los órganos colegiados superiores. Ahora bien, como el artículo 143,2 , contempla la existencia de una iniciativa autonómica cumulativa, en el supuesto que nos ocupa dicha iniciativa debe ser ejercida tanto por los. mencionados órganos colegiados superiores como por los Municipios. Por lo tanto, los organos preautonómicos no pueden ejercer por sí solos la iniciativa del proceso autonómico. Con ello; se limitan sus competencias en esta materia, pues tan sólo pueden sustituir a las Diputaciones u órganos interinsulares.

En general, son de aplicar aquí las mismas consideraciones que hemos expuesto al analizar el supuesto general. Sin embargo, se plantea la cuestión de si los plazos que establece el artículo 143 mantienen su vigencia en este supuesto. En principio, creo que el 
plazo de los seis meses no afecta para nada a este supuesto, porque los órganos colegiados superiores de los entes preautonómicos no admiten la calificación de Corporaciones locales ni tienen esta consideración en los respectivos Reales Decretos-leyes que los crean. Tampoco es aplicable el plazo de cinco años, pues la disposición transitoria séptima establece la posibilidad de disolución de los órganos preautonómicos si no ejerciesen la iniciativa autonómica en el plazo de tres años. Es decir, los órganos preautonómicos que ejerciesen dicha iniciativa nunca podrían, caso de que no prosperase, reiterarla pasados cinco años, pues con anterioridad a este plazo quedarían automáticamente disueltos. Con ello se obliga de algún modo a los órganos preautonómicos a ejercer la iniciativa autonómica, neutralizando así el carácter potestativo con que se les atribuye esta facultad por la disposición transitoria primera. Lo que sí afecta a este supuesto es lo que hemos dicho referente al aplazamiento de la iniciativa autonómica en virtud de lo dispuesto en la disposición transitoria tercera. Naturalmente, sólo afecta a los Municipios, pero como los órganos preautonómicos no pueden ejercer por sí solos la iniciativa autonómica, ya que se necesita también el concurso de los Municipios, resulta que esta disposición transitoria tercera también les afecta, si bien es verdad que no se producen consecuencias tan nefastas como en el caso del artículo 143, pues - como hemos dicho- aquí no cuenta el plazo de los seis meses. Es decir, los órganos preautonómicos podrían ejercer inmediatamente la iniciativa autonómica, pero tendríamos que esperar, por tratarse de una iniciativa cumulativa y no alternativa, a que la ejerciesen también los Municipios, una vez celebradas las primeras elecciones locales.

La disposición transitoria segunda también se refiere a los órganos preautonómicos. Sin embargo, aquí no se les atribuye la iniciativa del proceso autonómico, sino la iniciativa en el proceso de ampliación de competencias y la participación, mediante la convocatoria de la asamblea de parlamentarios, en la elaboración del Estatuto de Autonomía en los supuestos del artículo 151, 2. Es decir, no se les atribuye el primer paso del proceso autonómico, cual es la iniciativa autonómica (de la cual son titulares, pero únicamente en virtud de lo dispuesto en la disposición transitoria primera), sino unos pasos o momentos posteriores y sucesivos del mencionado proceso autonómico. Por ello, creo que los órganos preautonómicos no pueden acogerse a esta disposición transitoria 
para ejercer la iniciativa autonómica, sino tan sólo para ejercer las atribuciones que expresamente les confiere y que acabamos de mencionar (10).

\section{LAS DiputacioneS, LOS ÓRgANOS INTERINSULARES Y LOS MUNICIPIOS COMO TITULARES ESPECIALES DE LA INICIATIVA AUTONÓMICA}

El artículo 151, 1, dispone: "No será preciso dejar transcurrir el plazo de cinco años a que se refiere el apartado 2 del artículo 148 cuando la iniciativa del proceso autonómico sea acordada dentro del plazo del artículo 143, 2, además de por las Diputaciones u órgano interinsulares correspondientes, por las tres cuartas partes de los Municipios de cada una de las Provincias afectadas que representen, al menos, la mayoría del censo electoral de cada una de ellas y dicha iniciativa sea ratificada mediante referéndum por el voto afirmativo de la mayoría absoluta de los electores de cada Provincia en los términos que establezca una Ley Orgánica». Este supuesto especial es el mismo que hemos estudiado en el supuesto general con una serie de cualificaciones exigidas para ejercer la iniciativa autonómica, cualificaciones que producen dos importantes consecuencias: la primera de ellas consiste en que en este caso las futuras Comunidades autónomas pueden asumir el mayor número de competencias posibles, respetando siempre el marco del artículo 149, que enumera las competencias exclusivas del Estado, sin necesidad de dejar transcurrir el plazo de cinco años a que se refiere el artículo 148; y la segunda, la necesidad de que en este supuesto el procedimiento para la elaboración del Estatuto no va a ser el procedimiento general establecido en el artículo 146, sino el establecido en el artículo 151, 2. Con ello, si la iniciativa autonómica se realiza de acuerdo con este precepto, se creará una nueva categoría de Comunidad autónoma, caracertizada por poseer un mayor número de competencias y por elaborar y aprobar el Estatuto de Autonomía con arreglo a un procedimiento específico. Pero dejemos estas cuestiones y analicemos los problemas que plantea este supuesto especial de iniciativa autonómica.

En principio, los titulares de dicha iniciativa son los mismos que en el supuesto general: Diputaciones, órganos interinsulares y

(10) Sobre esta cuestión y, en general, sobre los regimenes preautonómicos, cfr. mi trabajo aLa Constitución y los regímenes preautonómicos», de próxima publicación en la Revista de Estudios Politicos. 
Municipios. Las diferencias se refieren a que no se habla de atodas las Diputaciones interesadas», aunque el significado es el mismo, pues se requiere el acuerdo de todas las Diputaciones del ámbito territorial de referencia, y que en el caso de los Municipios se exige una cualificación mayor, pues los titulares son las tres cuartas partes de los Municipios. Pero quizá lo más destacado es que, con esta redacción, la existencia de la iniciativa cumulativa no admite ningún tipo de duda, exigiéndose que dicha iniciativa sea ejercida tanto por las Diputaciones u órganos interinsulares como por las tres cuartas partes de los Municipios. No otro significado tiene la expresión «además de».

En cuanto a los requisitos exigidos para ejercer dicha iniciativa, se plantean aquí semejantes cuestiones a las estudiadas en el supuesto general. Por ello, y para evitar repeticiones, vamos a centrarnos únicamente en el estudio de las diferencias. En principio, la iniciativa por parte de las tres cuartas partes de los Municipios va referida necesariamente a cada una de las Provincias afectadas. Es decir, no se trata de las tres cuartas partes de los Municipios de la totalidad del territorio afectado, sino de cada una de las Provincias afectadas, exigiéndose, además, que esas tres cuartas partes de los Municipios representen, al menos, la mayoría - se entiende necesariamente relativa- del censo electoral de cada Provincia. Son de aplicar aquí las consideraciones que hicimos en el supuesto general en torno a la expresión «censo electoral», así como el señalar que con esta redacción prácticamente se está confiriendo un derecho de veto a la cuarta parte más uno de los Municipios, sin tener en cuenta la población que representen. Aparte de exigir esta mayor cualificación - los tres cuartos-, desaparece toda referencia al término isla, que tantos inconvenientes presentaba. Asimismo se suprime la palabra «población» del artículo 143, 2, con lo cual se logra una mayor coherencia en la redacción del precepto, ya que los términos población y censo electoral, como vimos, podían encerrar ciertas contradicciones.

Además de estos requisitos, ahora se va a exigir que la iniciativa autonómica sea ratificada mediante referéndum. Este referéndum debe ser aprobado por "el voto afirmativo de la mayoría absoluta de los electores de cada Provincia». Aparte de que esto supone una nueva potenciación de la Provincia, lo grave es que, al exigirse el voto afirmativo de la mayoría absoluta de los electores, resulta que la iniciativa autonómica no solamente debe ser ratifi- 
cada por la mayoría absoluta - mitad más uno- (lo cual, de por sí, ya es una exigencia excesiva, máxime si se ha exigido anteriormente que la iniciativa deba ser ejercida por las tres cuartas partes de los Municipios) de la población, sino que, en realidad, se exige una cualificación mayor, pues no se trata de la mayoría absoluta de los votos válidamente emitidos - tal como señala, por ejemplo, el artículo $151,2,4 .^{\circ}$ y la disposición transitoria cuarta-, sino de la mayoría absoluta de los electores. Es decir, las abstenciones y los votos nulos juegan en contra de esa mayoría absoluta. Por todo ello, creo que se trata de una exigencia excesivamente rigurosa, y que el referéndum, caso de ser exigido (pues ya es suficiente con que la iniciativa tenga que ser ejercida por las tres cuartas partes de los Municipios), debería haber sido suficiente con que hubiera sido aprobado por la mayoría relativa de los votos válidamente emitidos.

En cuanto a los plazos para el ejercicio de dicha iniciativa, en principio es de aplicar, pues así se establece taxativamente, en este supuesto el plazo de los seis meses. Con ello, y debido al mayor número de requisitos exigidos en este supuesto, resulta muy difícil que la iniciativa autonómica pueda realizarse en ese exiguo plazo de tiempo. En cuanto al plazo de cinco años, en principio parece que no afecta para nada a este supuesto, ya que el precepto nos remite únicamente al plazo de seis meses. Así pues, dicha iniciativa, caso de que no prospere, puede reiterarse en cualquier momento, sin necesidad de que transcurran cinco años. También pudiera interpretarse que el plazo de los cinco años tiene aquí plena vigencia, pues el supuesto general (art. 143) es supletorio, en cuanto a los requisitos exigidos, de este supuesto especial, máxime si se trata de los mismos titulares de la iniciativa autonómica.

Tampoco es de aplicar a este supuesto el contenido de la disposición transitoria tercera, ya que su redacción es terminante, pues sólo se refiere a la iniciativa de las Corporaciones locales prevista en el artículo 143, 2, y no, aunque sean las mismas, a las del artílo 151, 1. Ello puede parecer un contrasentido, pues las Corporaciones locales del artículo 151, 1, son las mismas que las del artículo $143,2, y$, por consiguiente, contienen, en su composición y funcionamiento, los mismos defectos de ausencia de democratización. Quizá, pues parece imposible que se trate de un olvido de los autores de la Constitución, se pensó que esta exigencia era innecesaria en el supuesto que nos ocupa, ya que la iniciativa de las Corpora- 
ciones locales debía ser ratificada mediate referéndum, lo cual, en algún modo, paliaba esa falta de democracia de las citadas Corporaciones. El argumento no parece suficiente, pues, aunque el referéndum pueda cumplir, en algún modo, dicha función, sin embargo, la iniciativa autonómica no corresponde a los electores, que únicamente ratificaban dicha iniciativa, sino a las Corporaciones locales citadas, las cuales, evidentemente, carecen de cualquier elemento democrático.

Aunque, como acabamos de ver, la disposición transitoria tercera no paraliza la iniciativa autonómica en este supuesto especial, sin embargo dicha iniciativa puede ser paralizada, o al menos retrasada, estableciéndose con ello un obstáculo más que haría más difícil -prácticamente imposible- el cumplimiento del plazo de los seis meses, ya que se exige que el referéndum ratificatorio de la iniciativa autonómica debe ser realizado en los términos que establezca una Ley Orgánica. Con ello, habrá que esperar a que las Cortes Generales aprueben la mencionada Ley Orgánica que regule las modalidades de este referéndum. Ello, indudablmente, retrasará o condicionará el ejercicio de la iniciativa autonómica en este supuesto especial que, como hemos visto, presenta ya de por sí suficientes dificultades para su ejercicio.

\section{SUPUESTOS PARTICULARES}

Como supuestos particulares de los titulares de la iniciativa del proceso autonómico, vamos a analizar dos casos, merecedores de esta calificación: el de Navarra y los de Ceuta y Melilla, supuestos que vienen contemplados, precisamente por su espectacularidad, en las disposiciones transitorias.

\section{La intciativa aUtonomica en el Caso de NavarRa}

La disposición transitoria cuarta establece: «1. En el caso de Navarra, y a efectos de su incorporación al Consejo General Vasco o al régimen autonómico vasco que le sustituya, en lugar de lo que establece el artículo 143 de la Constitución, la iniciativa corresponde al órgano foral competente, el cual adoptará su decisión por mayoría de los miembros que lo componen. Para la validez de dicha iniciativa será preciso, además, que la decisión del órgano foral competente sea ratificada por referéndum expresamente convoca- 
do al efecto y aprobado por la mayoría de los votos válidamente emitidos. 2. Si la iniciativa no prosperase, solamente se podrá reproducir la misma en distinto período de mandato del órgano foral competente, y en todo caso cuando haya transcurrido el plazo mínimo que establece el artículo 143». Este supuesto particular nos plantea una serie de consideraciones.

La iniciativa autonómica no va a corresponder a las Diputaciones y Municipios, como sucedía en el supuesto general, sino al organo foral competente, es decir, a la Diputación foral de Navarra. Ahora bien, esta iniciativa únicamente corresponderá a la citada Diputación foral cuando se trate de la incorporación de Navarra al Consejo General Vasco o al régimen autonómico vasco que le sustituya. Pero si se trata de la configuración de Navarra como Comunidad autónoma, con independencia del País Vasco, sería de aplicar el supuesto general establecido en el artículo 143, suponiendo que Navarra fuera considerada como una Provincia con entidad regional histórica (art. 143, 1), para lo cual no parecen existir excesivos problemas; o bien se aplicaría, en el supuesto de que no se produjese esta consideración, lo establecido en el artículo 144, a), que ya hemos analizado. Aquí, pues, únicamente vamos a considerar el supuesto particular de la incorporación de Navarra al País Vasco.

Una vez determinado quién es el titular de la iniciativa autonómica, la Diputación foral de Navarra, veamos el procedimiento y los requisitos exigidos para que dicha iniciativa prospere. En principio, dicha iniciativa debe ser adoptada por acuerdo de los miembros que componen la referida Diputación, acuerdo adoptado por mayoría, se entiende simple o relativa. Un avez acordada la iniciativa autonómica, se requiere, para su validez, que sea ratificada, mediante referéndum expresamente convocado, por la mayoría, se entiende simple o relativa, de los votos válidamente emitidos. En principio, los requisitos no parecen excesivos, máxime si se comparan con los exigidos para otros supuestos que hemos analizado. Quizás el problema más importante que se plantea, en cuanto a la interpretación del texto se refiere, es el de determinar quién debe convocar el referéndum y con qué requisitos y condiciones debe éste celebrarse. La disposición transitoria que estamos analizando guarda absoluto silencio, estableciendo únicamente que el referéndum debe ser expresamente convocado al efecto. Ante este silencio, hemos de acudir al artículo 62, c), que atribuye al Rey la facultad 
de convocar a referéndum en los casos previstos en la Constitución -y éste es uno de ellos-, atribución que deberá ser refrendada por el Presidente del Gobierno (arts. 56, 3, y 64, 1). Junto a ello, el artículo 92, 3, señala que una Ley Orgánica «regulará las condiciones y el procedimiento de las distintas modalidades de referéndum previstas en la Constitución». Así pues, para ejercer dicha iniciativa autonómica o, mejor dicho, para que ésta pueda prosperar, es necesario esperar a la promulgación de la citada Ley Orgánica, lo cual, evidentemente, retrasa el ejercicio de dicha iniciativa. De todas formas, parece indudable que el referéndum deberá ser convocado expresamente por el Rey, con el refrendo del Presidente del Gobierno.

Si esta iniciativa autonómica, para cuyo ejercicio no se establece ningún plazo, ni siquiera el de los seis meses previsto en el artículo 143, 2, no prosperase, podrá reiterarse - la Constitución habla de "reproducirse»-, siempre que se cumplan dos condiciones necesarias: que haya transcurrido el plazo de cinco años establecido en el artículo 143, 3, y que, además, nos encontremos en presencia de un período de mandato distinto del órgano foral competente, lo cual, evidentemente, puede alargar sobremanera el período de los cinco años.

\section{La iniciativa autonómica en los casos de CeUta y Melilla}

A tal efecto, la disposición transitoria quinta señala: «Las ciudades de Ceuta y Melilla podrán constituirse en Comunidad autónoma si así lo deciden sus respectivos Ayuntamientos, mediante acuerdo adoptado por la mayoría absoluta de sus miembros y así lo autorizan las Cortes Generales, mediante una Ley Orgánica, en los términos previstos en el artículo $144 »$. Este supuesto particular del ejercicio de la iniciativa autonómica tiene lugar, pues, cuando las ciudades de Ceuta y Melilla pretendan acceder a la condición de Comunidades autónomas, pretensión que no tiene por qué necesariamente realizarse, pues el texto habla únicamente de "podrán», es decir que tiene un carácter potestativo.

Los titulares de la iniciativa autonómica son los respectivos Ayuntamientos de ambas ciudades, que deberán tomar su decisión mediante acuerdo adoptado por la mayoría absoluta de sus miembros. En principio, no son de aplicar aquí los plazos establecidos en el artículo 143, sobre todo el plazo de los seis meses. En cuanto 
al plazo de los seis años, pudiera entenderse que sí es aplicable, ya que en el caso de que esta iniciativa no prosperase podría ser reiterada pasados los cinco años que establece el artículo 143 . De todas formas, ante el silencio constitucional, me inclino por la primera de las soluciones apuntadas, pudiéndose, por tanto, reiterar la iniciativa autonómica en cualquier momento, sin necesidad de esperar a que transcurran cinco años. Tampoco es de aplicar aquí lo establecido en la disposición transitoria tercera. Es decir, los Ayuntamientos de Ceuta y Melilla pueden ejercer dicha iniciativa sin esperar a que se celebren las primeras elecciones locales, una vez vigente la Constitución. Ello parece plantear las mismas incorrecciones que hemos expuesto al analizar el artículo 151, 1, pues los Ayuntamientos de ambas ciudades carecen también del más elemental carácter democrático.

Ahora bien, esta iniciativa de los Ayuntamientos - para cuyo ejercicio no se exigen demasiados requisitos- no es suficiente, sino que se exige, además, que sea autorizada por las Cortes Generales, mediante una Ley Orgánica, por motivos de interés nacional. Es decir, esta autorización hay que ponerla en relación con el artículo 144 , a), al que ya nos hemos referido. Ahora bien, esta autorización de las Cortes Generales no debe, en ningún momento, ser entendida como una auténtica iniciativa autonómica, ya que los únicos sujetos de ésta son los Ayuntamientos de ambas ciudades. Evidentemente, el papel atribuido a las Cortes es trascendental, pues, aparte de poder autorizar o no la iniciativa autonómica ejercida por los Ayuntamientos, pueden, sin necesidad de tomar ninguna postura formal al respecto, impedir o al menos retrasar considerablemente - mediante cualquier tipo de dilaciones y obstrucciones- la iniciativa autonómica. Esta, al menos, se verá retrasada hasta la aprobación de la correspondiente Ley Orgánica. Sin duda, con ello, se puede provocar un enfrentamiento entre las Cortes Generales y los Ayuntamientos de Ceuta y Melilla. Y todo ello sin olvidar el contexto internacional que es necesario tener presente siempre en este caso.

\section{LA POSTURA DE LAS FUERZAS POLITICAS}

En líneas generales, las fuerzas políticas, tanto en el Congreso como en el Senado, prestaron atención suficiente al tema de la iniciativa autonómica, siendo éste uno de los más debatidos dentro 
del problema general de las autonomías. Normalmente, participan en los debates la mayoría de las fuerzas políticas. Sin embargo, el análisis de los debates parlamentarios resulta insuficiente, pues algunas fuerzas políticas no participan en ellos, expresando su postura a través de las enmiendas presentadas a los diferentes preceptos, por lo cual su estudio se nos hace indispensable por ser la única fuente de conocimiento para exponer su postura. A fin de lograr un conocimiento global, haremos un estudio por separado, tratando de evitar repeticiones, entre la postura de las fuerzas políticas del Congreso y las del Senado.

A) En el Congreso de los Diputados, la postura de las fuerzas políticas puede resumirse así:

UCD interviene activamente en los debates sobre esta cuestión. Aunque no se reservó ningún voto particular en el dictamen de 5 de enero, presentó diversas enmiendas, tanto a título de grupo parlamentario como sus miembros individualmente, logrando la redacción definitiva de varios preceptos o incorporando otros al texto constitucional a través de las enmiendas in voce. En líneas generales, se muestra partidaria de exigir fuertes requisitos para el ejercicio de la iniciativa autonómica, aunque alguno de sus miembros pretendía la reducción de tales requisitos (11).

El PSOE tampoco se reservó ningún voto particular al citado dictamen de 5 de enero. Posteriormente presentó diversas enmiendas al supuesto general, consiguiendo introducir la disposición transitoria tercera y modificar la redacción del artículo 151, 1. En los debates mantiene posturas no muy distantes de UCD (12), aunque interviene menos activamente.

Los Socialistas de Cataluña mantienen las mismas tesis y posturas que el PSOE. No intervienen en los debates y su papel se reduce a presentar alguna enmienda y a apoyar las intervenciones de los socialistas (13).

(11) Vid. enmiendas de los señores Garcfa-Margallo (núms. 752 y 753), Bravo de Laguna (núm. 767), Martínez Pujalte (núm. 743), Ortí Bordás (núm. 736), Pérez Crespo (núm. 68), Astrain (núm. 778 bis) y de UCD como grupo (núm. 778). Vid., asimismo, las enmiendas in voce del señor Meilán Gil (D. S. Congreso, sesión del 14 de junio, págs. 3255 y sigs.), otra defendida por el mismo diputado (D. S. Con. greso, sesión del 15 de junio, págs. 3310-3311) y otra del señor García-Margallo (D. S. Congreso, sesión del 20 de junio, págs. 3510-3511).

(12) Vid. enmienda núm. 350, en la que se atribuía la iniciativa autonómica también a diputados y senadores, y la 778 sobre Navarra, firmada también por UCD y PNV. Vid., asimismo, enmiendas in voce del señor Zapatero (D. S. Congreso, sesiones de 16 y 20 de junio, págs. 3410 y sigs.).

(13) Vid. enmienda núm. 302, que coincide exactamente con la 350 del grupo socialista. 
El PCE, por su parte, aunque presentó ya un voto particular en el dictamen de 5 de enero, no interviene en los debates ni presenta ningún tipo de enmiendas, a excepción de los temas referentes a regímenes preautonómicos (14).

AP sí interviene en los debates. Se reservó diversos votos particulares al dictamen de 5 de enero, que posteriormente defendió en los debates, tratando de restringir los titulares de la iniciativa autonómica y exigir el mayor número posible de garantías para su ejercicio (15).

Aunque la Minoría Catalana no interviene en estos debates, sin embargo el PNV presenta sus enmiendas, interviniendo posteriormente cuando se trata de la cuestión navarra y en el tema de los regímenes preautonómicos (16).

Finalmente, el Grupo Mixto es, junto con UCD, el que más activamente interviene, sin duda debido al carácter heterogéneo de sus componentes. Presentó diversas enmiendas, tratando de favorecer la iniciativa autonómica mediante la supresión del mayor número posible de requisitos, aunque sin lograr nunca sus objetivos (17).

B) En el Senado los debates mantienen, en líneas generales, la misma tónica que en el Congreso. Las enmiendas e intervenciones se centran sobre los requisitos y procedimiento exigido para el ejercicio de la iniciativa autonómica. Normalmente, las fuerzas políticas que más intervienen son aquellas que no tienen representación en el Congreso y, por las mismas razones que en la Cámara Baja, el Grupo Mixto.

UCD presenta, aunque no grupo parlamentario, sino sus miembros a título individual, diversas enmiendas que van desde la su-

(14) Vid. voto particular PCE al artículo 129,1 , del texto de 5 de enero $(B . O . C$., número 44), en el cual se atribuye la iniciativa autonómica a los Ayuntamientos. Vid., asimismo, las intervenciones del señor Solé Tura (D. S. Congreso, sesión del 15 de junio, págs. 3411 y sigs.), logrando la redacción definitiva de la disposición transitoria primera.

(15) Vid. voto particular de AP al artículo 129 del texto de 5 de enero, donde se establecen determinados requisitos gravosos. Vid., asimismo, las intervenciones del señor Fraga (D. S. Congreso, sesión del 14 de junio, págs. 3251-3252) y del señor Carro (D.S. Congreso, sesión del 16 de junio, pág. 3412 ) sobre los regímenes preautonómicos.

(16) Vid. la ya citada enmienda núm. 778 sobre Navarra y las intervenciones del señor Vizcaya (D. S. Congreso, sesión del 2 de junio, págs. 3509-3510).

(17) Vid. las enmiendas de los señores Gómez de las Roces (núm. 56), Letamendía (núm. 64), Gastón Sanz (núm. 84) y Sánchez Ayuso (núm. 565). Vid. especialmente las intervenciones de los señores Gómez de las Roces $(D$. S . Congreso, se. sión del 14 de junio, págs. 3252-3253, y del 18 de julio, págs. 4387-4391); Letamendía (D. S. Congreso, sesión del 14 de junio, pág. 3253) y Gastón Sanz (D. S. Congreso, sesión del 14 de junio, pág. 3254, así como sus reiteradas intervenciones en el Pleno del Congreso en las sesiones de 18,19 y 21 de jullo). 
presión de los requisitos gravosos para el ejercicio de la iniciativa autonómica, hasta la constitucionalización del derecho de separación, pasando por determinadas correcciones gramaticales y de estilo y por regular de una forma más detallada los supuestos particulares de Navarra, Ceuta y Melilla. En los debates no se discuten todas las enmiendas, siendo las intervenciones de menor entidad que las del Congreso (18).

El PSOE, por su parte, también presentó alguna enmienda a paliar, en algún modo, los plazos exigidos en el supuesto general y a corregir defectos gramaticales. En los debates interviene, aunque no con frecuencia, en la defensa de sus enmiendas (19).

El PSI (Progresistas y Socialistas Independientes) presentó diversas enmiendas encaminadas a suprimir los requisitos gravosos para el ejercicio de la iniciativa autonómica, procuando establecer un único procedimiento en el ejercicio de dicha iniciativa, así como aumentar las atribuciones de los órganos preautonómicos. Aunque también interviene en los debates, no logra nunca ningún resultado positivo (20).

La Agrupación Independiente (AI) también presentó diversas enmiendas tendentes a efectuar correcciones terminológicas y limar los requisitos exigidos en el artículo 151, 1, efectuando en los debates diversas interpretaciones sobre los inconvenientes que presentan los diferentes preceptos (21).

El Grupo Independiente (GI) también interviene en estos debates a través de una serie de enmiendas tendentes a la supresión de las Cortes como titulares de la iniciativa autonómica, no interviniendo en los debates, a excepción del caso de Navarra (22).

(18) Vid. las enmiendas de los señores Ballarin (núms. 930 y 950), Angulo Montes (núm. 931), Galván González (núms. 932 y 949), Leira (núm. 974) y Burgo Tajadura (núms. 974 y 975). Son de destacar, asimismo, las intervenciones de los señores Angulo Montes ( $D$. S. Senado, sesión del 12 de septiembre, págs. 25432544), Galván (D. S. Senado, sesión del 12 de septiembre, págs. 2548-2550) y Burgo Tajadura (D. S. Senado, sesiones del 14 de septiembre y 5 de octubre, págs. 2761 y 3348, respectivamente).

(19) Vid. las enmiendas núms. 1.083 y 1.088, así como las intervenciones del señor Sainz de Varanda (D. S. Senado, sesión del 12 de septiembre, pág. 2546, y sesión del 13 de septiembre, pág. 2653).

(20) Vid. las enmiendas núm. 81, 84, 87, 98 y 99, asf como las intervenciones de los señores Martín-Retortillo (D. S. Senado, sesiones del 12 y 14 de septiem. bre, págs. 2544 y 2757, respectivamente), López Martos (D. S. Senado, sesión del 3 de octubre, págs. 3224-3227) y Villar Arregui (D. S. Senado, sesiones del 12 y 14 de septiembre, págs. 2551 y 2756 , respectivamente).

(21) Vid. las enmiendas núms. 629, 635 y 650, asi como las intervenciones del señor Ollero (D. S. Senado, sesión del 12 de septiembre, págs. 2546-2548, y sesión del 12 de septiembre, págs. 2653-2655).

(22) Vid. las enmiendas núms. 379, del señor Prado Leiria, y 386, del señor Díz Alegria, así como las intervenciones de este último senador sobre el caso de 
El Grupo de Senadores Vascos también presenta alguna enmienda con el fin de evitar las discriminaciones en materia de iniciativa autonómica, interviniendo en los debates únicamente en turno de portavoces para explicar su postura en las diversas votaciones (23).

Entesa dels Catalans, por su parte, únicamente interviene cuando se trata de la regulación de los órganos preautonómicos como titulares de la iniciativa autonómica (24).

Finalmente, el Grupo Mixto presenta diversas enmiendas que, debido a su heterogeneidad, van desde la total supresión del tema relativo a las autonomías, hasta la supresión de la iniciativa autonómica por parte de las Cortes Generales, sin olvidar las presentadas para establecer un Estado confederal y limitar los requisitos exigidos en el supuesto general (25).

Del análisis efectuado sobre estos debates parlamentarios, podemos extraer las siguientes conclusiones:

a) El tema de la iniciativa autonómica suscitó suficiente atención por parte de las fuerzas políticas presentadas en ambas Cámaras.

b) La mayoría de las enmiendas e intervenciones se centraron en los requisitos exigidos para ejercer la iniciativa autonómica, encontrándose las posturas de quienes mantenían un número excesivo de requisitos y las de quienes pretendían evitar obstáculos para dicho ejercicio.

c) Tan sólo dos fuerzas políticas, UCD y PSOE, lograron hacer triunfar sus posturas, funcionando de esta forma el llamado «consenso». En efecto, UCD logra la redacción definitiva de los artículos 143 y 144 y disposiciones transitorias cuarta y quinta. Por su parte, el PSOE logra la redacción definitiva del artículo 151, 1, y de la disposición transitoria tercera. A ello hay que añadir que también el PCE logró la redacción definitiva de la disposición tran. sitoria primera.

Navarra (D. S. Senado, sesiones del 14 de septiembre y 5 de octubre, págs. 2766 y 3353-3354, respectivamente).

(23) Vid. enmienda núm. 310, del señor Bandrés, y las intervenciones de los señores Unzueta (D. S. Senado, sesión del 12 de septiembre, pág. 3550) y Monreal (D. S. Senado, sesiones del 14 de septiembre y 5 de octubre, págs. 27642765 y 3351 3352 , respectivamente).

(24) Vid. enmiendas núms. 807 y 808 , así como las intervenciones del señor Benet en su defensa ( $D$. S. Senado, sesión del 14 de septiembre, págs. 2758 y sigs.).

(25) Vid. enmiendas núms. 226, del señor Carazo; 284 y 279, del señor Zarazaga; $554,568,569$ y 570 , del señor Xirinacs. Vid., asimismo, la intervención del señor Zarazaga (D. S. Senado, sesión del 12 de septiembre, pág. 2595). 